\title{
Life Table Study of Tobacco Caterpillar Spodoptera litura (F.) (Noctuidae: Lepidoptera) on Different Bt Cotton Hybrids During 120-150 DAS (Days after Sowing)
}

\author{
G.B. Supriya ${ }^{1} *$, T.V.K. Singh ${ }^{1}$, V. Sunitha ${ }^{2}$, S.K. Vinod ${ }^{3}$ and C. Narendrareddy ${ }^{1}$ \\ ${ }^{1}$ Department of Entomology, College of Agriculture, Prof. Jayashankar Telangana State \\ Agricultural University, Rajendranagar, Hyderabad - 500 030, India \\ ${ }^{2}$ All India Network Project on Vertebrate Pest Management, PJTSAU, Rajendranagar, \\ Hyderabad - 500 030, India \\ ${ }^{3}$ ICRISAT, Patancheru, Hyderabad, India \\ *Corresponding author
}

\begin{tabular}{|c|c|c|}
\hline & \multicolumn{2}{|l|}{ A B S T R A C T } \\
\hline $\begin{array}{l}\text { S. litura, Bt- cotton, } \\
\text { Age-specific } \\
\text { survivorship, } \\
\text { Fecundity and life } \\
\text { expectancy }\end{array}$ & \multirow{3}{*}{\multicolumn{2}{|c|}{$\begin{array}{l}\text { Survival and fertility characteristics of the third instar larvae of Spodoptera litura } \\
\text { (Fabricius), on Bt-I and Bt-II cotton hybrids during } 120-150 \text { DAS were assessed in the } \\
\text { laboratory. Life tables and population parameters of the S.litura were constructed in an } \\
\text { environment with unlimited food supply and that was free of natural enemies. The results } \\
\text { revealed that the modest rate of mortality occurred in the immature stage, especially in the } \\
\text { third instar. The population parameters calculated are recorded as low in Bt-II hybrids } \\
\text { compared to Bt-I hybrids. The net reproductive rate }\left(\mathrm{R}_{0}\right) \text { recorded highest } 447.24 \text { on NCS- } \\
954 \text { Bt-I whereas lowest on RCH-134 Bt-II as } 169.35 \text { with the mean generation time (Tc) } \\
40.01 \text { days. Intrinsic rate of increase }\left(\mathrm{r}_{\mathrm{m}}\right) \text { was ranged from } 0.12-0.18 \text {, the highest } \mathrm{r}_{\mathrm{m}} \text { was } \\
\text { recorded on Bt-I hybrids. The population doubling time (DT) was } 5.39 \text { days on RCH-134 } \\
\text { Bt-II. The Bt-II hybrids have shown a profound impact on the growth and development of } \\
\text { S.litura during early stages of crop, as the crop matured the Cry toxin content decreased, } \\
\text { survival rate recorded was more in } 4^{\text {th }} \text { and } 5^{\text {th }} \text { instar larvae in later stages of crop. }\end{array}$}} \\
\hline Article Info & & \\
\hline & & \\
\hline Introduction & & \\
\hline \multicolumn{3}{|c|}{$\begin{array}{lll}\text { Modern biotechnology is dramatically } & \text { advantages over traditional chemical } \\
\text { redefining pest management in global cotton } & \text { insecticides. Cotton hybrids expressing Cry } \\
\text { production. After a decade of research, } & \text { proteins are grown worldwide for the } \\
\text { transgenic, insect-resistant cotton varieties } & \text { management of Lepidopteran pests. However, } \\
\text { were developed that enable growers to use an } & \text { in crops with several target pests with varying } \\
\text { in-plant protection method as part of their } & \text { degrees of susceptibility to Bt (e.g., cotton), } \\
\text { integrated pest management programs (Perlak } & \text { there is concern regarding the suboptimal } \\
\text { et al., 1990). The use of transgenic crops production of toxin, resulting in reduced }\end{array}$} \\
\hline
\end{tabular}


efficacy and increased risk of Bt resistance during later stages of crop.

The fall in expression with advancement of season or age of the plant has been well documented in genotypes expressing Cry1 Ac in cotton and many other genes in other crops. The decline in expression has been reported in fruiting structure (Greenplate 1999, Adamczyk et al., 2001, Horwitz et al., 2003, Kranthi et al., 2005, Wan et al., 2005).

Dual toxins (Cry1Ac+Cry2Ab) provided substantially better control of $H$. zea, S. frugiperda and $S$. exigua than single toxin Cry1Ac (Stewart et al., 2001). Quite a few reports are also available for BG-II events stating decline in expression, however the dual genes have been able to contain the pest significantly (Udikeri, 2006 and Somashekar, 2009).

Due to the wide adoption of Bt transgenic crops, the efficacy of this technology is threatened by the evolution of resistance by target pests. After more than a decade of commercialization, recent reports support field-evolved resistance to $\mathrm{Bt}$ crops in Helicoverpa zea (Tabashnik et al 2008), Spodoptera frugiperda (Storer et al 2010), Busseolafusca (Van Rensburg, 2007), and Spodoptera litura (Jeya Kumar et al., 2007).

Spodoptera litura (Fabricius) is the cosmopolitan insect species. It is belongs to Phylum -Arthropoda, Subphylum -Uniramia, Order -Lepidoptera, Family- Noctuidae and Subfamily- Amphipyrinae. It is an extremely serious pest, the larvae can defoliate many economically important crops. It is seasonally common in annual and perennial agricultural systems in tropical and temperate Asia. This noctuid is often found as part of a complex of Lepidopteran and non-Lepidopteran foliar feeders but may also injure tubers and roots. Hosts include field crops grown for food and fibre, plantation and forestry crops, as well as certain weed species (CABI, 2010).

The main objective of the experiment was to know the survivorship, fecundity, intrinsic rate stable age distribution and life expectancy of third instar larvae of S.litura on different Bt cotton hybrids during 120-150DAS (Days after sowing). For this life table was constructed on each hybrid. Life table studies are fundamental to population ecology.

The cohort life table gives the most comprehensive description of the survivorship, development and reproduction of a population that are fundamental factors in both theoretical and applied population ecology (Taghizadeh et al., 2008). Life tables are of a great value in understanding the population dynamics of a species because they provide an integrated view of the biological characteristics of a given population under certain environmental conditions (Coppel and Mertins 1977). Although the present study was conducted under controlled conditions, insects in nature experience adverse biotic and abiotic factors that affect their fitness, so that life table studies are useful in evaluating these eco-logical factors (Nava et al 2008).

The life table parameters, particularly the intrinsic rate of natural increase $\left(\mathrm{r}_{\mathrm{m}}\right)$, are the most important parameters that can be used to evaluate the level of plant resistance to insects (Razmjouet al., 2006). Host plants displaying lower values of $r_{m}$ are relatively more resistant than the plants with higher values of $r_{m}$. In the present study, the life table parameters, especially the $r_{m}$, are used to compare the potential population growth of S.litura on single and dual gene Bt cotton hybrids.

The present study is an effort to fulfil the deficiency of data on survival of third instar larvae of S.litura on Bt-I and Bt-II hybrids. Therefore, the present experiment provides 
novel information on the life table parameters of third instar larvae of S.litura on single and dual gene Bt cotton hybrids.

\section{Materials and Methods}

Laboratory experiments were carried out during 20122013 to study the effect of transgenic Bt cotton hybrids consisting single and double toxins leaves against ofS.litura. The seeds of different Bt cotton hybrids as mentioned below were obtained from Monsanto Seed Company, Hyderabad.Seeds were dibbled with intra row spacing of $60 \mathrm{~cm}$ and inter row spacing of $90 \mathrm{~cm}$. In each treatment plot, 100 plants were maintained. Leaves of 60 days old crop were used in present experimentation.

\begin{tabular}{|l|l|l|l|}
\hline \multicolumn{2}{|c|}{ Bt-I HYBRIDS } & \multicolumn{2}{c|}{ Bt-II HYBRIDS } \\
\hline 1. & NCS-145 Bt-I & 7. & NCS-145 Bt-II \\
\hline 2. & NCS-207 Bt-I & 8. & NCS-207 Bt-II \\
\hline 3. & NCS-950 Bt-I & 9. & NCS-950 Bt-II \\
\hline 4. & NCS-954 Bt-I & 10. & NCS-954 Bt-II \\
\hline 5. & RCH-2 Bt-I & 11. & RCH-2 Bt-II \\
\hline 6. & RCH-134 Bt-I & 12. & RCH-134 Bt-II \\
\hline
\end{tabular}

\section{Insect colony}

A colony of S.litura was originally collected from a Colocasia fields at ARI (Agriculture Research Institute) Rajendranagar and maintained in the Bt-Lab, Department of Entomology, Professor Jayashankar Telangana State Agriculture University, Rajendranagar, Hyderabad. The colony was periodically supplemented with larvae collected from the different fields of castor and tomato to reduce inbreeding depression. The egg mass after hatching were transferred on to sorghum leaf based artificial diet (Kranthi, 2005). The grown up larvae were allowed to pupate in the soil. Moths were collected on emergence and released in battery jars for egg laying.

\section{Life table study}

The leaves of 120-150 DAS collected from field were washed with distilled water and sandwiched between two blotting papers to remove excess moisture from the leaf, and maintained in battery jars $(10 \times 15 \mathrm{~cm})$ with round disc of blotting paper at bottom of the jar. Larvae hatched from one cohort of eggs reared initially on artificial diet up to third instar were transferred to leaves of different Bt-I and Bt-II cotton hybrids of 90-120 DAS. The battery jars were closed at the top with a muslin cloth for ventilation and larvae were maintained in three replications till the death or pupation. The methods suggested by Morris and Miller (1954) were used for constructing the life-tables.

Adult moths that emerged from the larvae reared on different Bt-I and Bt-II cotton hybrids, collected with the help of plastic tube were released into battery jar, which was closed at the top with a muslin cloth for ventilation and the internal walls were covered with the white paper as an oviposition substrate. A small cotton wick soaked in 10 per cent honey solution was placed in the small petriplates for adult feeding. The number of eggs laid by the female adults on each day was counted by using stage microscope till the death of the adults. The life-table for female was constructed from the column $1 \mathrm{x}$ as described by Birch (1948) and Poole (1974). Stable age distribution was worked out by observing the population schedule of birth rate and death rate $(\mathrm{mx}$ and lx) when grown in limited space. Life expectancy was computed by using the method suggested by Deevey (1947) and Atwal and Bains (1974).

\section{Data analysis}

A computer software package MS-EXCEL was used for analysis.

\section{Results and Discussion}

At global scenario, life table statistics and intrinsic rates of increase have been studied by sizable workers in Lepidopteran insect pests. 
The contribution of Morris and Miller (1954) on Choristoneurafumiferana, Stark (1959) on Recurvastarki and LeRouxet al., (1963) on Spilonotaocellana are prominent.

\section{Age specific survivorship}

The age specific survivorship (lx) and mortality (dx) of $S$. litura on different Bt- I and Bt-II hybrids are presented in figures (112). When the larva fed with the Bt-I hybrids the survivorship curve observed was type I as the mortality is more in adult stages. Fig (1-6) (Slobodkin, 1962). Survival of third instar was low when reared on Bt-II hybrids. The pattern of survivorship that we observed indicated that the young and immature stage was more susceptible to physical disruptions caused by the suitability of the food quality. The survivorship curve indicated a modest rate of mortality during the early life stages and a gradual decrease as it approached later stages and this curve assumed a near type IV survivorship curve, (Slobodkin, 1962) it means most of the mortality is happening at the beginning of life cycle, i.e. mortality of third instar. Fig (7-12).

\section{Age specific fecundity}

The age specific female survivorship (lx) recorded highest in Bt-I hybrids compared to Bt-II hybrids. The lowest female survivorship was recorded in RCH-134 Bt-II as 0.19 whereas highest was recorded in 0.32 in $\mathrm{RCH}-$ 134 Bt-I (Fig.13-24). Potential fecundity $\left(\mathrm{m}_{\mathrm{x}}\right)$ was recorded more in larvae fed on Bt-I hybrids compared to Bt-II hybrids. Fecundity is defined as the number of offspring produced by an individual insect, whereas fertility is the number of viable offspring produced. Highest fecundity was recorded on NCS-207 Bt-I (1212.00) the lowest fecundity was observed on NCS-207 Bt-II (774.50).

\section{Population growth parameters}

The different life parameters calculated for $S$. litura on Bt-I and Bt-II cotton hybrids of has been presented in Table 1 . The lowest net reproductive rate $\left(\mathrm{R}_{0}\right)$ of $S$. litura was recorded on Bt-II (RCH-134 Bt-II) hybrids compared to Bt-I. Similar trend was recorded with regard to other life parameters like potential fecundity (PF), intrinsic rate of increase $\left(r_{m}\right)$, finite rate of increase $(\lambda)$ corrected generation time $(\mathrm{T})$, hypothetical $\mathrm{F}_{2}$ females, annual rate of increase (ARI) and weekly multiplication rate (WMR). The higher intrinsic rate of increase was recorded on Bt-I hybrids, It is due to the faster development of immature stages shorter generation time, higher survivorship and higher fecundity rates. High value of $r_{m}$ indicates the susceptibility of a host plant to insect feeding, while a low value of $r_{m}$ indicates that the host plant species is resistant to the pest.

\section{Stable age distribution and life expectancy}

The stable-age distribution of S.litura on Bt-I and Bt-II showed more than 90 per cent of immature stages contributed more than mature stages. (Table.2) Life expectancy of S.litura on Bt-I hybrids was more in early stages and declined gradually, Whereas the larvae fed on Bt-II hybrid leaves the life expectancy was more in early days but less compared to Bt-I hybrid fed larvae and declined gradually.(Table.3) 


\section{Int.J.Curr.Microbiol.App.Sci (2018) 7(6): 2367-2386}

Table.1 Life parameters of $S$. litura fed on different Bt Cotton Hybrids. 120-150 DAS (Days after sowing)

\begin{tabular}{|c|c|c|c|c|c|c|c|c|c|c|c|c|}
\hline & $\begin{array}{c}\text { NCS-145 } \\
\text { Bt-I }\end{array}$ & $\begin{array}{c}\text { NCS-145 } \\
\text { Bt-II }\end{array}$ & $\begin{array}{c}\text { NCS-207 } \\
\text { Bt-I }\end{array}$ & $\begin{array}{c}\text { NCS-207 } \\
\text { Bt-II }\end{array}$ & $\begin{array}{c}\text { NCS-950 } \\
\text { Bt-I }\end{array}$ & $\begin{array}{c}\text { NCS-950 } \\
\text { Bt-II }\end{array}$ & $\begin{array}{c}\text { NCS-954 } \\
\text { Bt-I }\end{array}$ & $\begin{array}{c}\text { NCS-954 } \\
\text { Bt-II }\end{array}$ & $\begin{array}{c}\text { RCH-2 } \\
\text { Bt-I }\end{array}$ & $\begin{array}{c}\text { RCH-2 } \\
\text { Bt-II }\end{array}$ & $\begin{array}{c}\text { RCH-134 } \\
\text { Bt-I }\end{array}$ & $\begin{array}{c}\text { RCH-134 } \\
\text { Bt-II }\end{array}$ \\
\hline Net reproductive rate $\left(\mathbf{R}_{0}\right)$ & 364.72 & 233.79 & 478.73 & 192.71 & 383.30 & 233.69 & 447.24 & 210.09 & 349.37 & 268.40 & 285.63 & 169.35 \\
\hline $\begin{array}{l}\text { Mean duration of a generation } \\
\text { (Tc) (days) }\end{array}$ & 33.05 & 39.01 & 33.21 & 39.07 & 34.12 & 38.90 & 33.10 & 38.00 & 32.01 & 38.10 & 34.00 & 40.01 \\
\hline Arbitrary ' $r_{\mathrm{m}}$ ' or ' $r_{\mathrm{c}}$ ' value & 0.1784 & 0.1398 & 0.1858 & 0.1346 & 0.1743 & 0.1402 & 0.1844 & 0.1407 & 0.1829 & 0.1467 & 0.1662 & 0.1282 \\
\hline $\begin{array}{l}\text { Innate capacity for increase in } \\
\text { number }\left(\mathbf{r}_{\mathrm{m}}\right)\end{array}$ & 0.1791 & 0.1401 & 0.1864 & 0.1349 & 0.1748 & 0.1404 & 0.1849 & 0.1410 & 0.1835 & 0.1471 & 0.1667 & 0.1284 \\
\hline Potential fecundity & 1039.50 & 880.00 & 1212.00 & 774.50 & 1018.00 & 888.00 & 1175.00 & 819.00 & 1045.50 & 931.00 & 1117.00 & 885.50 \\
\hline $\begin{array}{l}\text { Finite rate of increase }(\lambda) \\
\qquad \varphi^{\prime} s / \varphi / \text { day }\end{array}$ & 1.19 & 1.15 & 1.20 & 1.14 & 1.19 & 1.15 & 1.20 & 1.15 & 1.20 & 1.15 & 1.18 & 1.13 \\
\hline $\begin{array}{l}\text { Corrected generation time (T) } \\
\text { (days) }\end{array}$ & 32.92 & 38.93 & 33.10 & 39.00 & 34.02 & 38.83 & 32.99 & 37.09 & 31.91 & 38.00 & 33.90 & 39.94 \\
\hline $\begin{array}{l}\text { Weekly multiplication rate } \\
\qquad\left(\mathrm{e}^{\mathrm{rm}}\right)^{7}\end{array}$ & 3.50 & 2.66 & 3.68 & 2.57 & 3.40 & 2.67 & 3.65 & 2.68 & 3.61 & 2.80 & 3.21 & 2.45 \\
\hline Hypothetical $\mathrm{F}_{2}$ females & 133020.67 & 54657.76 & 229182.41 & 37137.14 & 146918.89 & 54611.01 & 200023.61 & 44137.80 & 122059.39 & 72038.56 & 81584.49 & 28679.42 \\
\hline Doubling time (DT) & 3.86 & 4.94 & 3.71 & 5.13 & 3.96 & 4.93 & 3.74 & 4.91 & 3.77 & 4.71 & 4.15 & 5.39 \\
\hline Annual rate of increase (ARI) & $\begin{array}{c}2.5302 \times \\
10^{28}\end{array}$ & $\begin{array}{c}1.6154 \mathrm{x} \\
10^{22}\end{array}$ & $\begin{array}{c}3.5718 \mathrm{x} \\
10^{29}\end{array}$ & $\begin{array}{c}2.4210 \mathrm{x} \\
10^{21}\end{array}$ & $\begin{array}{c}5.2150 \mathrm{x} \\
10^{27}\end{array}$ & $\begin{array}{c}1.8376 \mathrm{x} \\
10^{22}\end{array}$ & $\begin{array}{c}2.0947 \mathrm{x} \\
10^{29}\end{array}$ & $\begin{array}{c}2.2850 \mathrm{x} \\
10^{22}\end{array}$ & $\begin{array}{c}1.2289 \mathrm{x} \\
10^{29}\end{array}$ & $\begin{array}{l}2.1253 \mathrm{x} \\
10^{23}\end{array}$ & $\begin{array}{c}2.7477 \mathrm{x} \\
10^{26}\end{array}$ & $\begin{array}{c}2.3278 \mathrm{x} \\
10^{20}\end{array}$ \\
\hline
\end{tabular}


Int.J.Curr.Microbiol.App.Sci (2018) 7(6): 2367-2386

Table.2 Stable age distribution of S.litura on different Bt-I and Bt-II hybrid

\begin{tabular}{|c|c|c|c|}
\hline & PER CENT & [ON OH & STAGES \\
\hline HYBRIDS & Larval & Pupal & Adult \\
\hline NCS-145 Bt-I & 96.08 & 3.53 & 0.37 \\
\hline NCS-145 Bt-II & 96.38 & 3.24 & 0.36 \\
\hline NCS-207 Bt-I & 96.26 & 3.39 & 0.33 \\
\hline NCS-207 Bt-II & 96.57 & 3.05 & 0.36 \\
\hline NCS-950 Bt-I & 96.21 & 3.42 & 0.35 \\
\hline NCS-950 Bt-II & 97.19 & 2.58 & 0.22 \\
\hline NCS-954 Bt-I & 96.35 & 3.35 & 0.30 \\
\hline NCS-954 Bt-II & 96.70 & 2.96 & 0.32 \\
\hline RCH-2 Bt-I & 96.85 & 2.81 & 0.32 \\
\hline RCH-2 Bt-II & 97.51 & 2.14 & 0.34 \\
\hline RCH-134 Bt-I & 95.95 & 3.66 & 0.38 \\
\hline RCH-134 Bt-II & 97.25 & 2.46 & 0.28 \\
\hline
\end{tabular}




\section{Int.J.Curr.Microbiol.App.Sci (2018) 7(6): 2367-2386}

Table.3 Life- expectancy of S. litura on different Bt-cotton hybrids

\begin{tabular}{|c|c|c|c|c|c|c|c|c|c|c|c|}
\hline \multicolumn{2}{|c|}{ NCS-145 Bt-I } & \multicolumn{2}{|c|}{ NCS-145 Bt-II } & \multicolumn{2}{|c|}{ NCS-207 Bt-I } & \multicolumn{2}{|c|}{ NCS-207 Bt-II } & \multicolumn{2}{|c|}{ NCS-950 Bt-I } & \multicolumn{2}{|c|}{ NCS-950 Bt-II } \\
\hline $\mathbf{x}$ & $\mathbf{e}_{\mathbf{x}}$ & $\mathbf{x}$ & $\mathbf{e}_{\mathbf{x}}$ & $\mathbf{x}$ & $\mathbf{e}_{\mathbf{x}}$ & $\mathbf{x}$ & $\mathbf{e}_{\mathbf{x}}$ & $\mathbf{x}$ & $\mathbf{e}_{\mathbf{x}}$ & $\mathbf{x}$ & $\mathbf{e}_{\mathbf{x}}$ \\
\hline $1-3$ & 29.45 & $1-3$ & 28.19 & $1-3$ & 32.03 & $1-3$ & 28.79 & $1-3$ & 32.66 & $1-3$ & 28.64 \\
\hline $4-6$ & 26.73 & $4-6$ & 28.43 & $4-6$ & 29.96 & $4-6$ & 27.81 & $4-6$ & 30.94 & $4-6$ & 26.75 \\
\hline $7-9$ & 25.90 & $7-9$ & 26.35 & $7-9$ & 27.85 & $7-9$ & 26.59 & $7-9$ & 28.24 & $7-9$ & 24.55 \\
\hline $10-12$ & 24.89 & $10-12$ & 25.85 & $10-12$ & 24.85 & $10-12$ & 25.74 & $10-12$ & 25.24 & $10-12$ & 23.10 \\
\hline $13-15$ & 22.17 & $13-15$ & 23.47 & $13-15$ & 22.35 & $13-15$ & 24.66 & $13-15$ & 22.24 & $13-15$ & 20.86 \\
\hline 16-18 & 19.17 & $16-18$ & 21.36 & $16-18$ & 19.57 & $16-18$ & 21.97 & 16-18 & 19.90 & 16-18 & 19.05 \\
\hline $19-21$ & 16.59 & $19-21$ & 19.78 & $19-21$ & 16.77 & $19-21$ & 19.83 & $19-21$ & 16.90 & $19-21$ & 18.24 \\
\hline$\overline{22-24}$ & 13.59 & $22-24$ & 17.32 & $22-24$ & 13.93 & $22-24$ & 18.20 & $22-24$ & 14.23 & $22-24$ & 16.23 \\
\hline $25-27$ & 10.74 & $25-27$ & 14.80 & $25-27$ & 11.07 & $25-27$ & 15.45 & $25-27$ & 11.51 & $25-27$ & 13.90 \\
\hline $28-30$ & 7.74 & $28-30$ & 11.80 & $28-30$ & 8.17 & $28-30$ & 12.45 & $28-30$ & 8.96 & $28-30$ & 11.10 \\
\hline $31-33$ & 4.74 & $31-33$ & 9.11 & $31-33$ & 5.24 & $31-33$ & 9.79 & $31-33$ & 6.05 & $31-33$ & 8.25 \\
\hline $34-36$ & 2.91 & $34-36$ & 6.23 & $34-36$ & 3.36 & $34-36$ & 7.19 & $34-36$ & 3.54 & $34-36$ & 5.57 \\
\hline $37-39$ & 2.00 & $37-39$ & 3.96 & $37-39$ & 2.00 & $37-39$ & 4.19 & $37-39$ & 2.00 & $37-39$ & 3.50 \\
\hline- & $40-42$ & 2.64 & - & - & $40-42$ & 2.58 & - & - & $40-42$ & 2.29 & - \\
\hline- & $43-45$ & 2.00 & - & - & $43-45$ & 2.00 & - & - & $43-45$ & 2.00 & - \\
\hline
\end{tabular}


Int.J.Curr.Microbiol.App.Sci (2018) 7(6): 2367-2386

Table.3 Cont.

\begin{tabular}{|c|c|c|c|c|c|c|c|c|c|c|c|}
\hline \multicolumn{2}{|c|}{ NCS-954 Bt-I } & \multicolumn{2}{|c|}{ NCS-954 Bt-II } & \multicolumn{2}{|c|}{ RCH-2 BT-1 } & \multicolumn{2}{|c|}{ RCH-2BT-II } & \multicolumn{2}{|c|}{ RCH-134 Bt-I } & \multicolumn{2}{|c|}{ RCH-134 Bt-II } \\
\hline $\mathbf{x}$ & ex & $\mathbf{x}$ & ex & $\mathbf{x}$ & ex & $\mathbf{x}$ & ex & $\mathrm{x}$ & ex & $\mathrm{x}$ & ex \\
\hline $11-3$ & 30.77 & $1-3$ & 26.06 & $1-3$ & 26.54 & $1-3$ & 26.48 & $1-3$ & 29.93 & $1-3$ & 25.22 \\
\hline $4-6$ & 28.36 & $4-6$ & 25.44 & $4-6$ & 24.83 & $4-6$ & 26.82 & $4-6$ & 28.71 & $4-6$ & 24.52 \\
\hline $7-9$ & 25.91 & $7-9$ & 24.39 & $7-9$ & 22.58 & $7-9$ & 25.00 & $7-9$ & 26.29 & $7-9$ & 23.11 \\
\hline $10-12$ & 23.41 & $10-12$ & 25.13 & $10-12$ & 20.51 & $10-12$ & 25.85 & $10-12$ & 23.83 & $10-12$ & 22.00 \\
\hline $13-15$ & 20.41 & $13-15$ & 23.49 & $13-15$ & 18.39 & $13-15$ & 26.68 & $13-15$ & 21.59 & $13-15$ & 20.97 \\
\hline $16-18$ & 17.41 & $16-18$ & 21.14 & $16-18$ & 16.43 & $16-18$ & 24.08 & 16-18 & 19.29 & $16-18$ & 20.73 \\
\hline $19-21$ & 15.47 & $19-21$ & 19.05 & $19-21$ & 14.83 & $19-21$ & 21.45 & $19-21$ & 16.49 & $19-21$ & 19.02 \\
\hline $22-24$ & 12.78 & $22-24$ & 16.61 & $22-24$ & 12.20 & $22-24$ & 19.12 & $22-24$ & 13.67 & $22-24$ & 17.28 \\
\hline $25-27$ & 10.04 & $25-27$ & 14.36 & $25-27$ & 9.50 & $25-27$ & 16.42 & $25-27$ & 10.81 & $25-27$ & 14.57 \\
\hline $28-30$ & 7.13 & $28-30$ & 11.58 & $28-30$ & 6.61 & $28-30$ & 13.95 & $28-30$ & 8.04 & $28-30$ & 12.06 \\
\hline $31-33$ & 4.26 & $31-33$ & 9.11 & $31-33$ & 3.83 & $31-33$ & 11.17 & $31-33$ & 5.53 & $31-33$ & 10.66 \\
\hline $34-36$ & 2.52 & $34-36$ & 6.38 & $34-36$ & 2.66 & $34-36$ & 8.34 & $34-36$ & 3.45 & $34-36$ & 8.77 \\
\hline $37-39$ & 2.00 & $37-39$ & 3.96 & $37-39$ & 2.00 & $37-39$ & 5.34 & $37-39$ & 2.00 & $37-39$ & 6.33 \\
\hline & $40-42$ & 2.75 & & & $40-42$ & 2.93 & & & $40-42$ & 3.59 & \\
\hline & $43-45$ & 2.00 & & & $43-45$ & 2.00 & & & $43-45$ & 2.00 & \\
\hline
\end{tabular}

X: Pivotal age in days

$\mathrm{e}_{\mathrm{x}}$ : Expectation of further life 


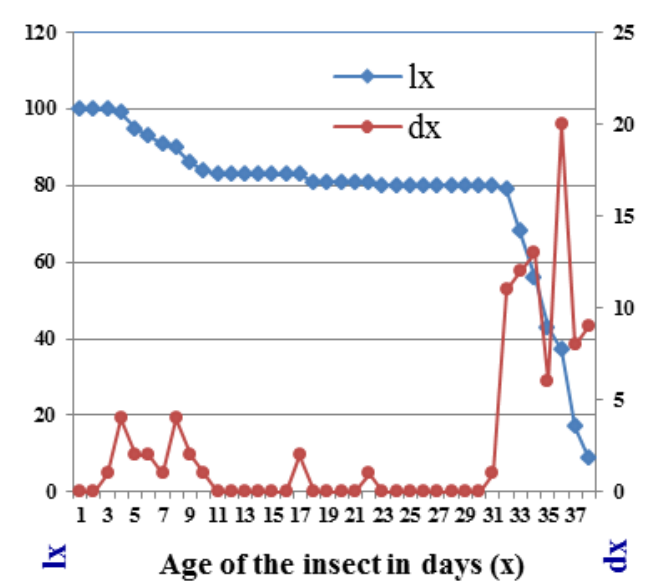

1. Age specific survivorship (lx) and mortality (dx) of S.litura on NCS-145 Bt-I

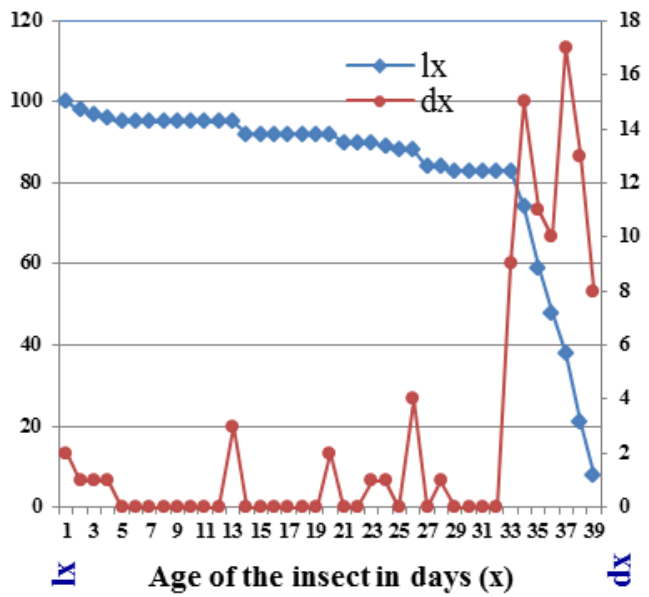

3.Age specific survivorship (lx) and mortality (dx) of S.litura on NCS-950 Bt-I

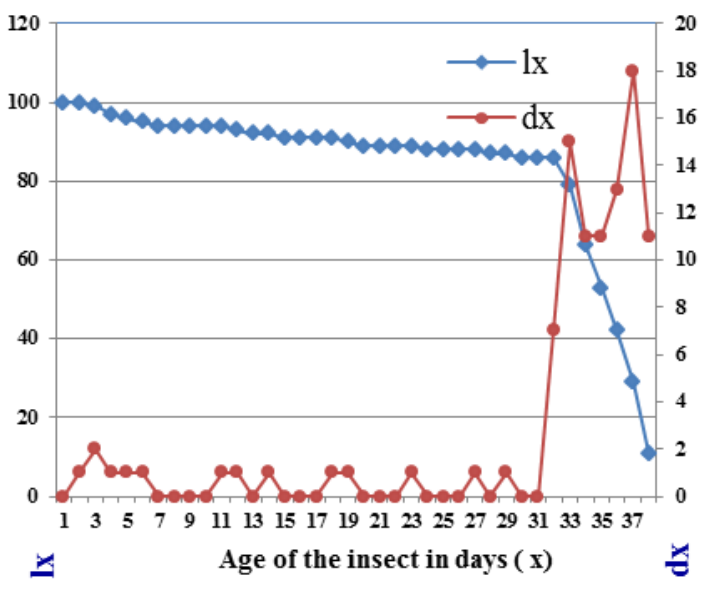

2. Age specific survivorship (lx) and mortality (dx) of S.litura on NCS-207Bt-I

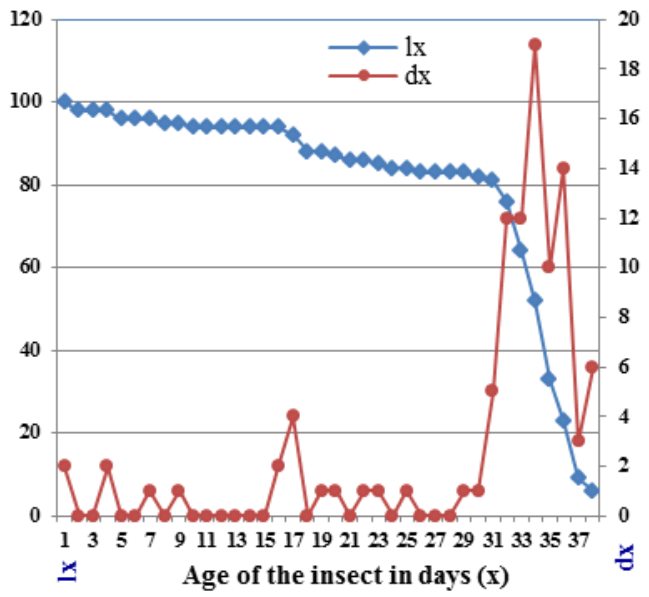

4. Age specific survivorship (lx) and mortality of S.litura on NCS954 Bt-I 


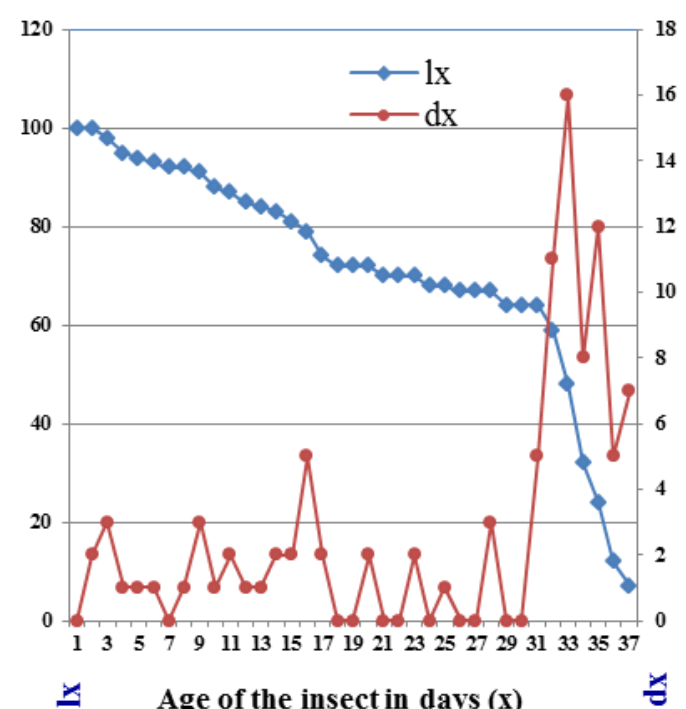

5.Age specific survivorship (lx) and mortality (dx) of S.litura on RCH-2Bt-I

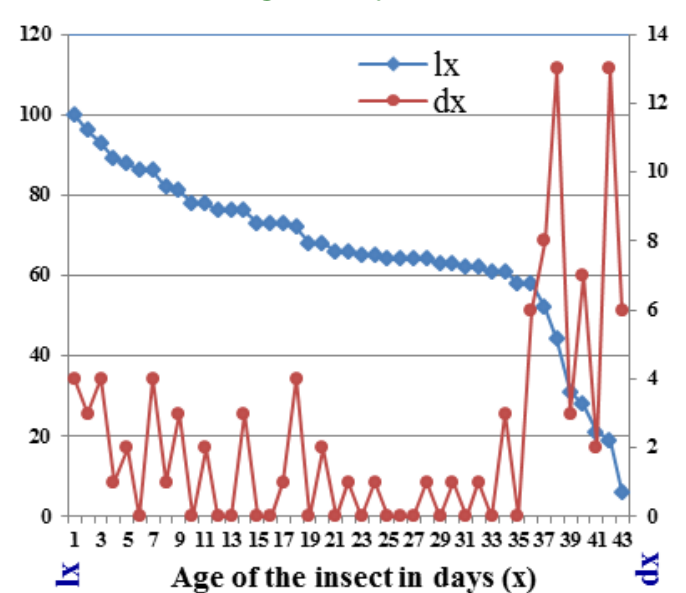

7. Age specific survivorship (lx) and mortality (dx) of S.litura on NCS-145 Bt-II

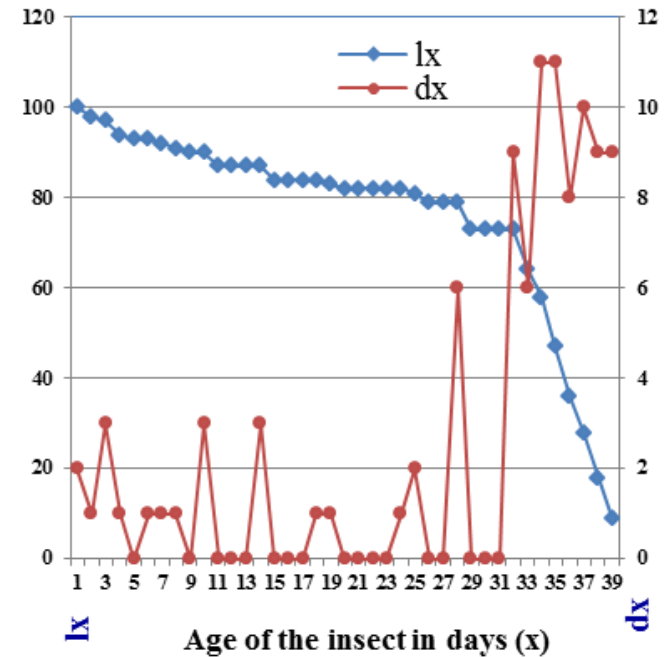

6. Age specific survivorship (lx) and mortality (dx) of S.lituraon RCH-134 Bt-I

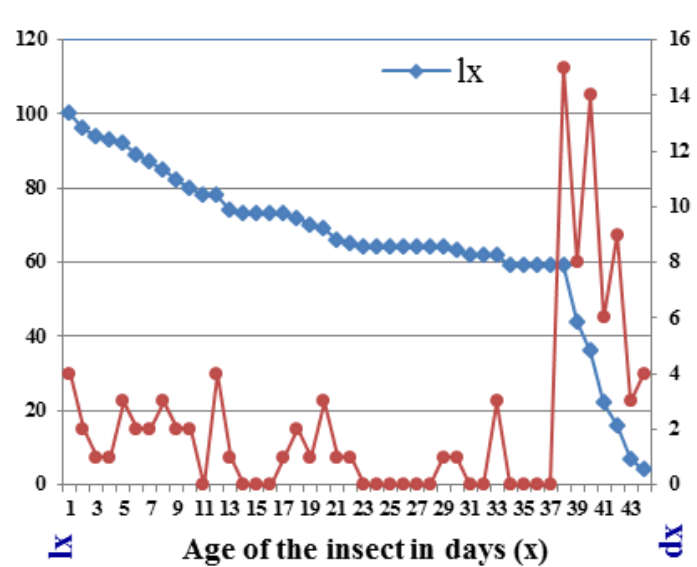

8.Age specific survivorship (lx) and mortality (dx) of S.litura on NCS-207 Bt-II 


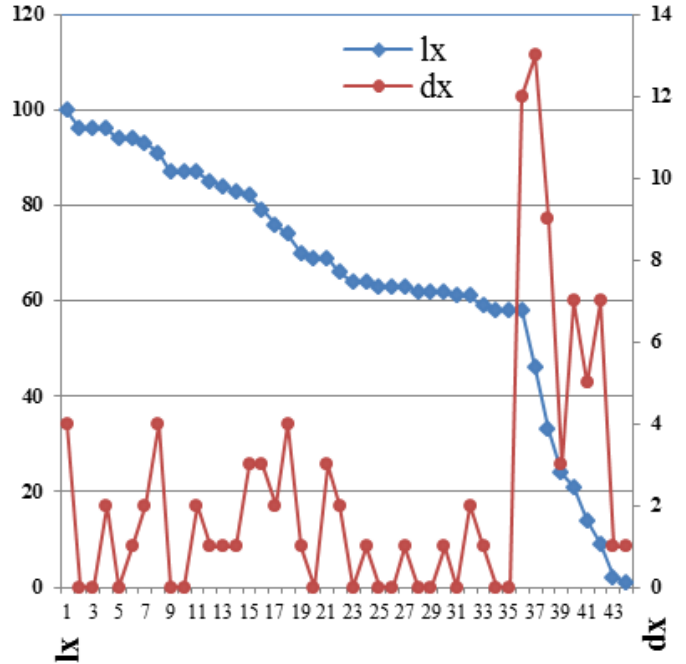

Age of the insect in days $(x)$

9. Age specific survivorship (1x) and mortality (dx) of S.litura on. NCS-950 Bt-II

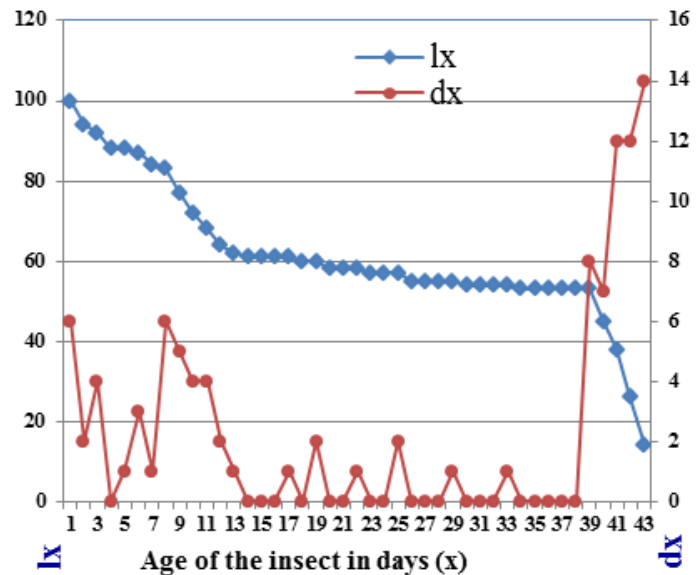

11.Age specific survivorship (lx) and mortality (dx) of S.litura on $\mathrm{RCH}-2$ Bt-II

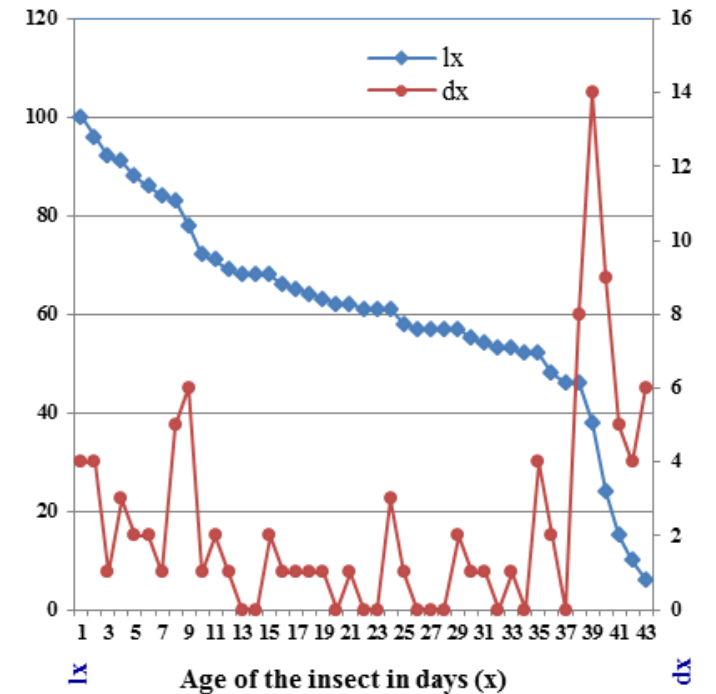

10. Age specific survivorship (lx) and mortality (dx) of S.litura on NCS-954 Bt-II

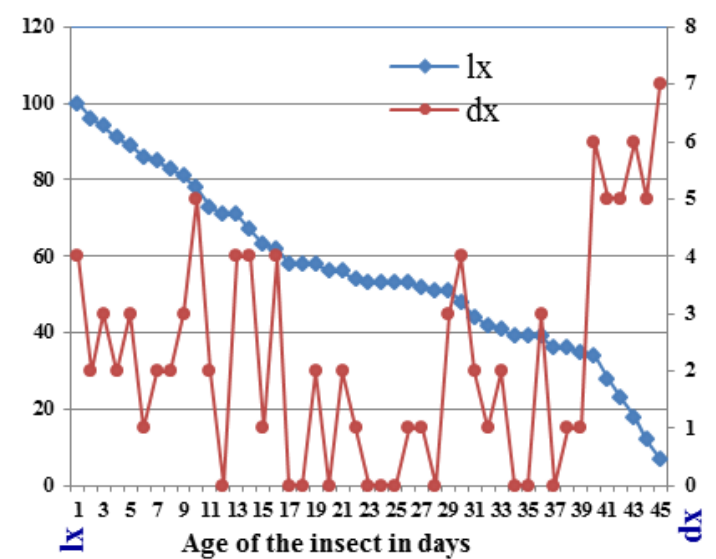

12. Age specific survivorship (lx) and mortality (dx) of S.litura RCH-134 Bt-II 


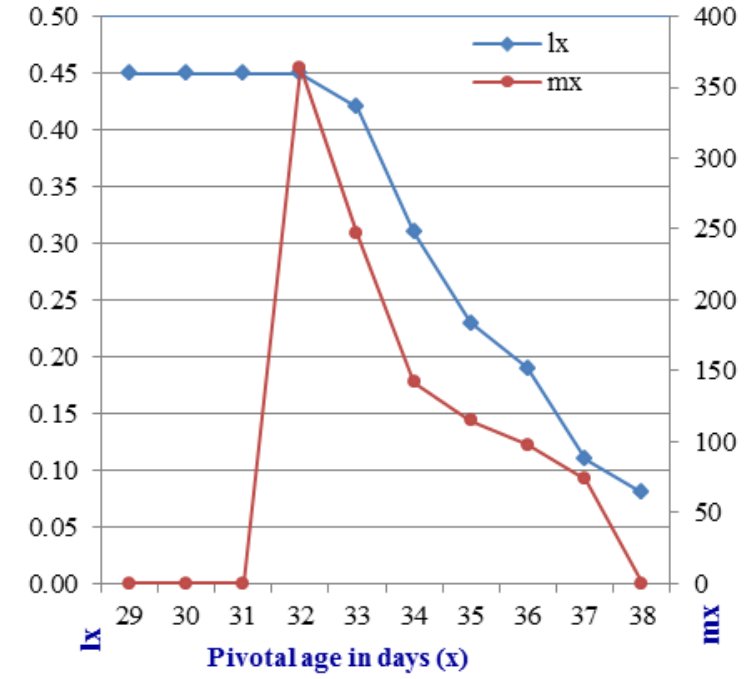

13. Age specific survivorship (lx) and fecundity (lx) of S.litura on NCS-145 Bt-I

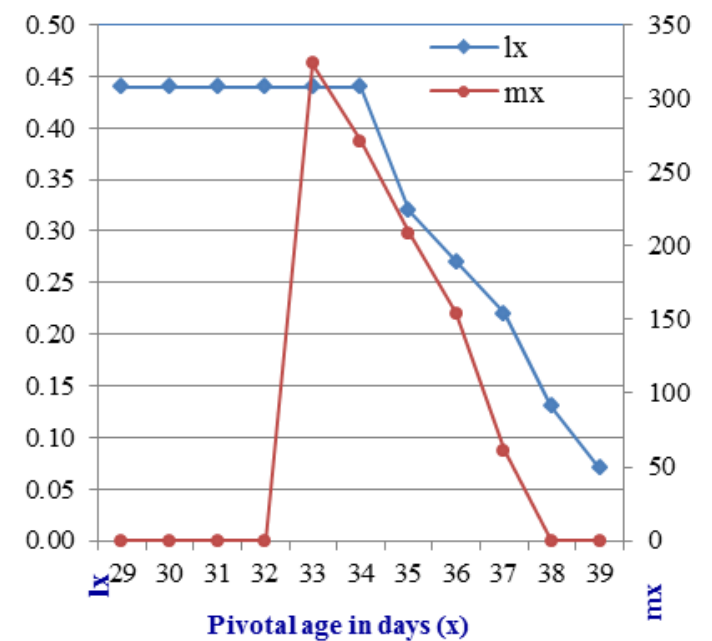

15. Age specific survivorship (lx) and fecundity (mx) of S.litura on NCS-950 Bt-I

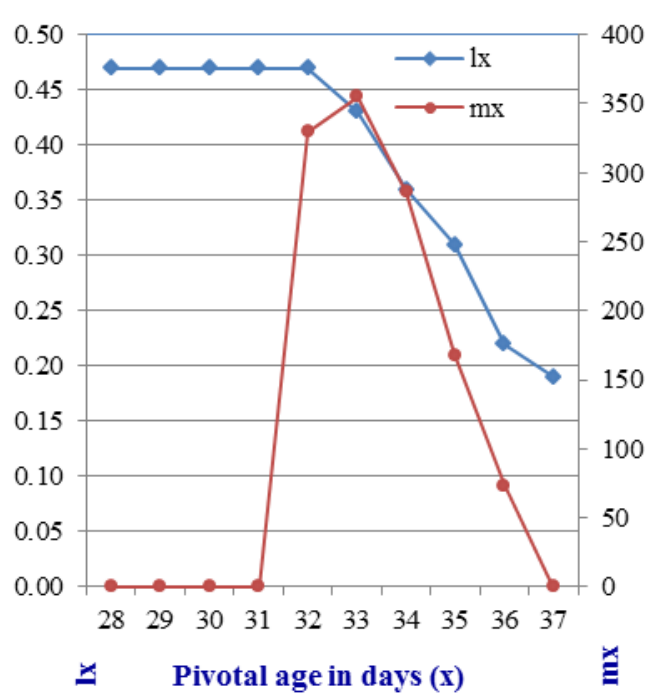

14. Age specific survivorship (lx) and fecundity (lx) of S.litura on NCS-207 Bt-I

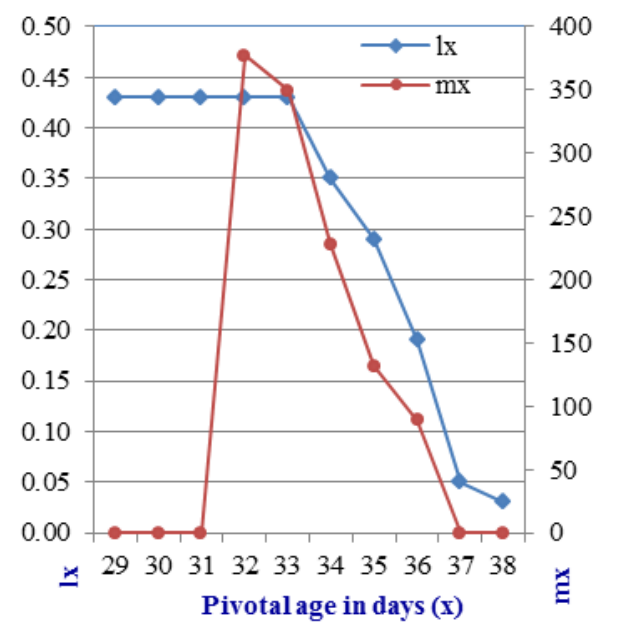

16.Age specific survivorship (lx) and fecundity (mx) of S.litura on NCS-954 Bt-I 


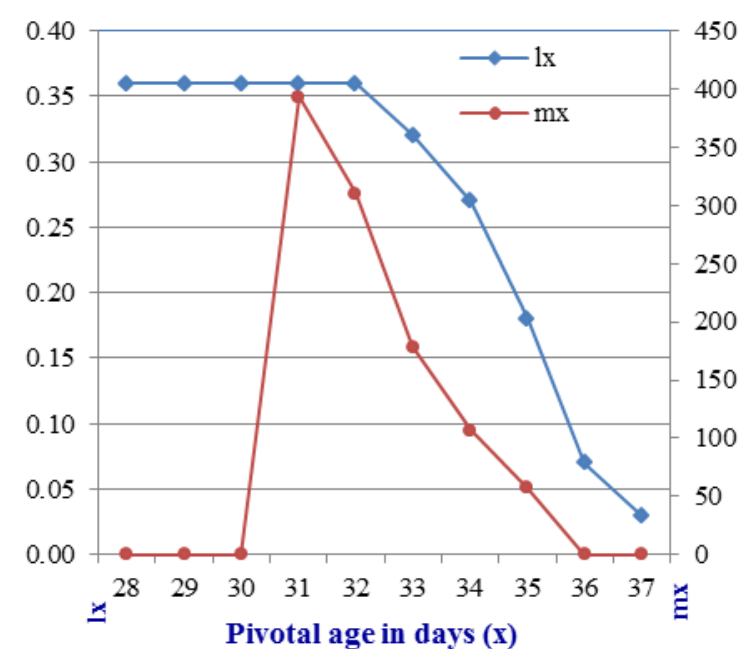

17. Age specific survivorship (lx) and fecundity (mx) of S.litura onRCH-2 Bt-I

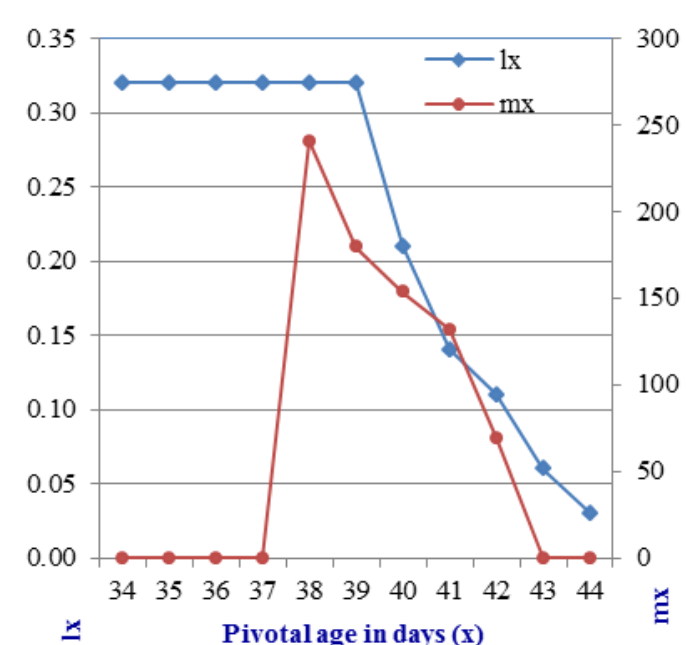

19. Age specific survivorship (1x) and fecundity (mx) of S.litura on NCS-145 Bt-II

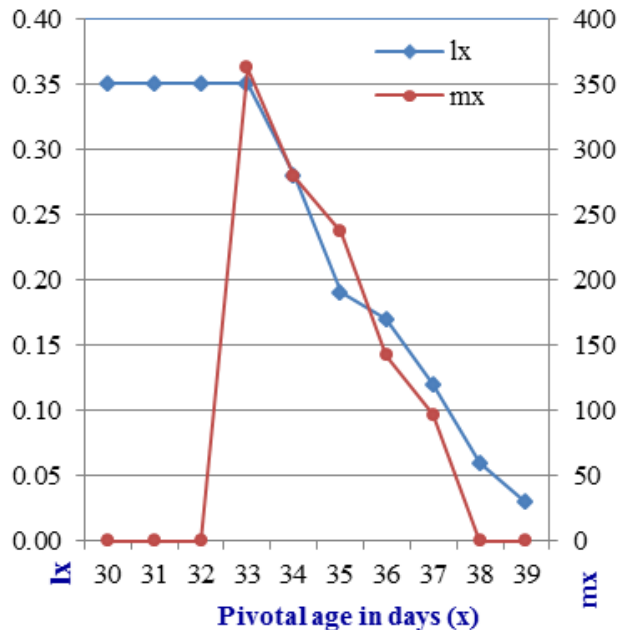

18.Age specific survivorship (lx) and fecundity (mx) of S.litura on RCH-134 Bt-I

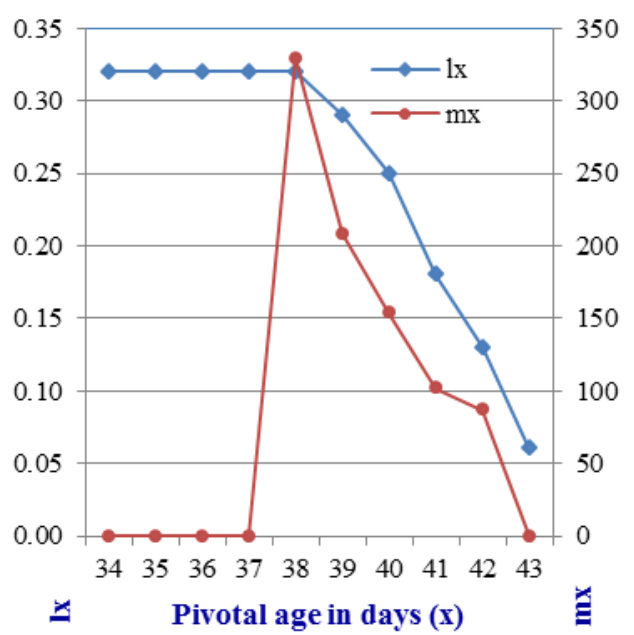

20. Age specific survivorship (lx) and fecundity (mx) of S.litura on NCS-207 Bt-II 


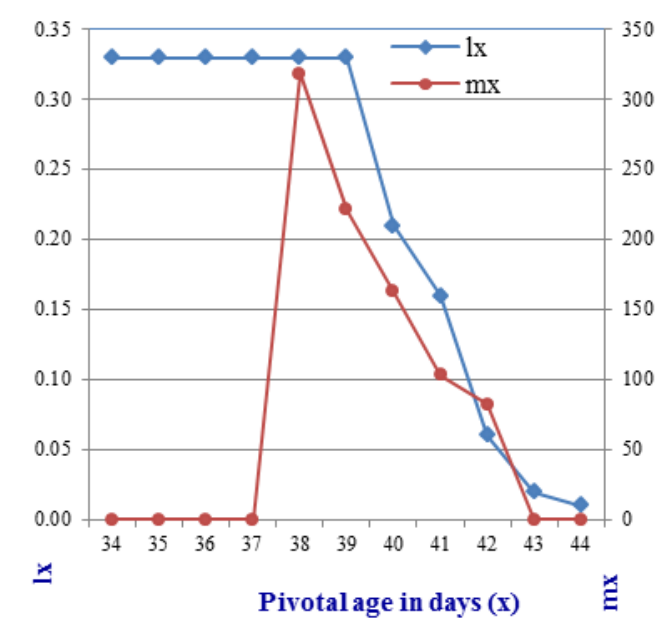

21. Age specific survivorship (lx) and fecundity (mx) of S.litura on NCS-950 Bt-II

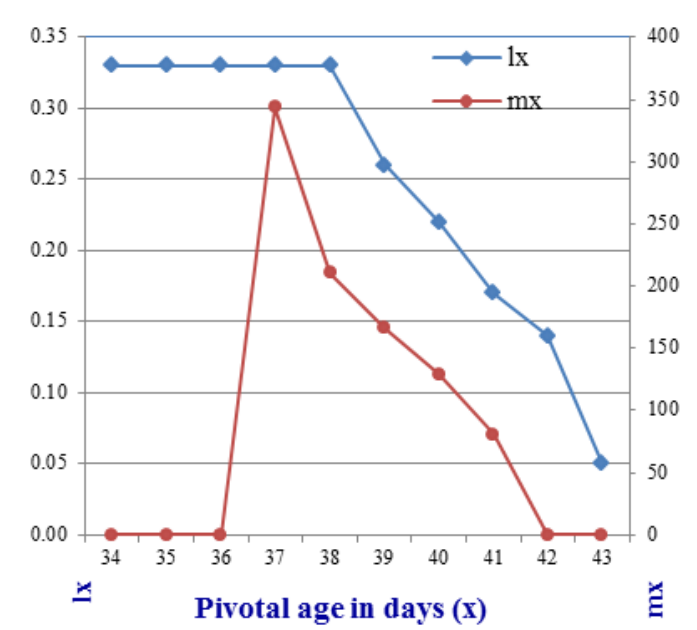

23. Age specific survivorship (lx) and fecundity (mx) of S.litura on $\mathrm{RCH}-2$ Bt-II

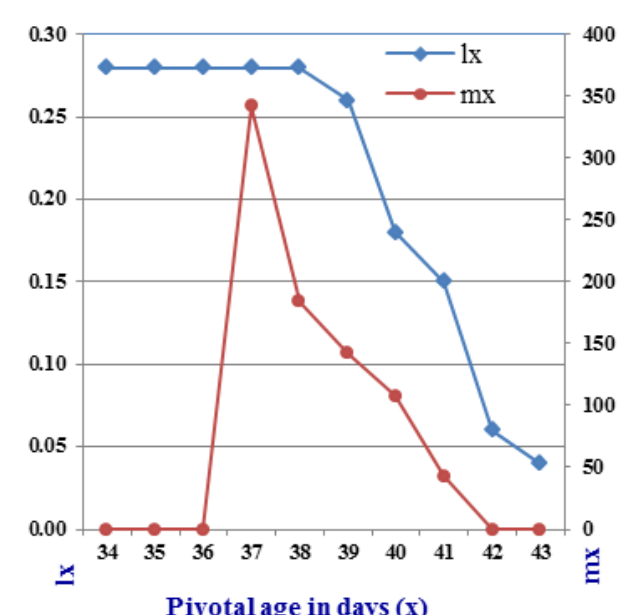

22. Age specific survivorship (1x) and fecundity (mx) of S.lituraon NCS-954 Bt-II

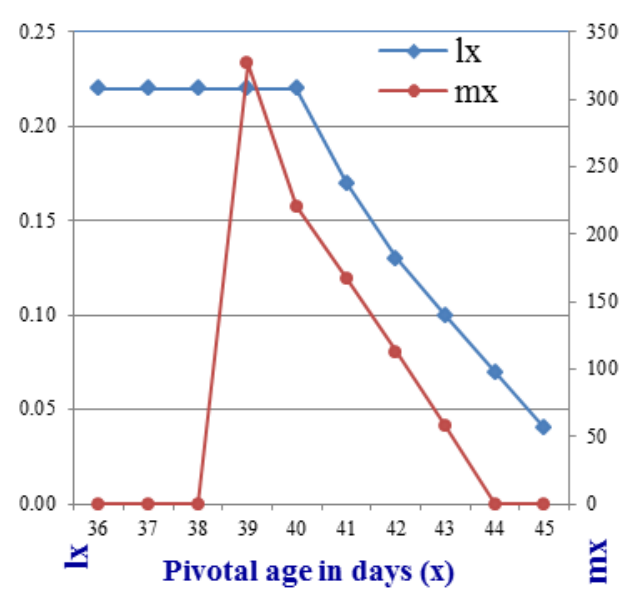

24.Age specific survivorship (lx) and fecundity (mx) of S.litura on RCH-134 Bt-II 


\section{Age specific survivorship}

The experiment (Generation-III) 120-150 DAS was started with the third instar larvae due to the higher mortality in the early stages during Generation -I (60-90 DAS) this can be supported with findings of authors Dhawan et al., (2010) they have reported that even at 120 DAS of the crop the mortality of neonates of S.litura fed on different plant parts of BG-II hybrids was recorded (from pooled analysis) more than 56.67- 80.00 per cent in leaves, 65.56-90.00 per cent in squares and 75.5683.33 per cent larval mortality on green bolls at ambient environmental condition. According to Kumar (2005), using the neonates of H.armigera, there was 89.26, 80.00 and 86.30 per cent mortality observed after 10 DPT (Days post treatment) period when the leaves, squares and bolls of 120 DAS was given to H.armigera, respectively. The results reported by Murugan et al., (2003) observed that first instar larvae of $H$. armigera when reared on the squares of transgenic cultivars showed highest mortality at 72 hours.

Large host range is considered important for better chance to survive during evolutionary strategies (Simpson et al., 2002; Raubenheimer and Simpson, 2003, Lee et al., 2003). Host plant range of generalist insect pests like $S$. litura may vary due to their higher level of feeding on different plant species and almost all parts of these plants (Schoonhoven et al., 1998; Suomela, 1996). Host selection may be associated with primary as well as secondary metabolites present in these plants which help them to choose preferred hosts due to nutritional variation (Ehrlich and Murphy, 1988; Rosenthal and Berenbaum, 1992, Simpson et al., 2002, Lee et al., 2003).

Insect herbivore relationship developed with respect to their feeding, survival and multiplication of generation. Such selection of different host plants helps in maintaining their numbers to multiply and maintain their diversity in nature (Raubenheimer and Simpson, 2003, Lee et al., 2003). This insect pest, as important general leaf feeder, utilizes green matter and in severe food shortage feeds on almost all parts of the plants. Such observations were clear when the leaves were either eaten up and transfer of insects to plant fruiting parts like flowers, fruits in different crops (Rosenthal and Berenbaum, 1992; Simpson et al., 2002). Host selection also depends on presence of plant metabolites which either attract or deter the pests (Ehrlich and Murphy, 1988, Hill, 1975). Cotton being the most suitable plant species is selected as a host by different sucking as well as chewing insect pests including S. litura. Presence of plant metabolites may also hinder their development but decrease deleterious effects due to gregarious feeding (Simpson et al., 2002, Lee et al., 2003).

\section{Age specific fecundity}

Potential fecundity may often be a good indicator of future reproductive output. In many Lepidopteran herbivore species all the eggs are present in the ovarioles at the adult moult, although they may not be all at the same stage of development, and host plant quality during larval growth and development is the key determinant of potential fecundity.

Adult insects need carbohydrate-rich food as their main source of energy for longevity, fecundity and mobility. The low number of eggs laid on a plant could have been affected by the more indirect route of reduced fecundity arising from larva feeding on nutritionally poor plants (Verkerk and Wright, 1996, Hamilton et al., 2005).

Host plant quality is a key determinant of the fecundity of herbivores insects, affecting 
insect reproductive strategies, egg size and quality, the allocation of resources to eggs, and the choice of oviposition sites may be influenced by plant quality, as may egg or embryo resorption on poor quality host (Awmack and Leather, 2002). Therefore, study of the influence of different host plants on the growth and development and fecundity of insects is very useful to understand host suitability of plant infesting insect species.

The morphological characteristics such as leaf shape, hairiness, bract shape and the presence or absence of nectar producing glands on leaves or flowers, and physical or structural qualities of host plant interfere with insect feeding behaviour. Maternal diet is considered an important factor for optimal insect growth (McIntyre and Gooding, 2000, Agrawal, 2001) and duration of offspring development (Roff, 1992).

\section{Population growth parameters}

The increase in growth rate, shorter development time and higher fecundity point out that increased feeding and or higher assimilation rate, both of which may be the result of an increased titer of digestive enzymes (Woods, 1999). Since intrinsic rate of increase $\left(r_{m}\right)$ is a reflective of many factors survival and generation time and adequately summarizes the physiological qualities of an animal in relation to its capacity to increase, it would be a most appropriate index to evaluate the performance of an insect on different host plants as well as the host plant's resistance (Kocourek et al., 1994; Southwood and Henderson, 2000). The intrinsic rate of increase $\left(r_{m}\right)$ is a more useful statistic to compare the population growth potential of different species than is $\mathrm{R}_{0}$ (Price, 1997). According to Southwood (1981) and Huffaker et al., (1984), r-strategists are characterized by a high $r_{m}$, a large fecundity (large $R_{0}$ ) and short generation time $(\mathrm{T})$.

\section{Stable age distribution}

The studies on stable-age distribution of the insect pest by different authors concluded that more than 90 per cent immature stages formed in the stable-age distribution (Bilapate and Pawar (1980), Bilapate (1980), Hemchandra and Singh (2003), Acharya et al., (2007), Gedia et al., (2008), and present results are in conformity with all of them. (Table.2)

\section{Life expectancy}

These results can be supported by findings of Dabhiet al., (2009) who reported that the life expectancy of P. xylostella was more in early stages and declined with advancement of age, similar results were reported by Patil et al (2014) of S.litura on tobacco.

In the Bt-II hybrids the life expectancy during 120-150 DAS was recorded as high during early days and decreased gradually, due to decline of Cry protein content in leaves during 120-150 DAS the third instar can able to feed on the leaves and Gilliland et al., (2002) reported that larvae become less susceptible to $\delta$-endotoxins as they age due to fewer binding sites in the older larvae.

The present results suggest that double gene hybrids had negative effect on the growth and development of S.litura during early stages of crop, as the crop matured the Cry toxin content decreased, the pest has developed resistance, hence the survival rate is more in fourth and fifth instar larvae in later stages of crop. It would be better to switch to Bt cotton that produces Cry $2 \mathrm{Ab}$ and one or more additional toxins unrelated to Cry1Ac, such as Vip3A (a vegetative insecticidal protein from $\mathrm{Bt}$ ), so that the increased frequency of resistance to Cry1Ac already seen in some populations would not reduce efficacy of the new Bt cotton. The future studies should 
elucidate on development of Bt cotton hybrids by using of refuge strategy to increase the susceptible population in the field. Plantspecific recommendations to reduce $\mathrm{Bt}$ resistance development include increasing $\mathrm{Bt}$ expression levels (high-dose strategy), expressing multiple toxins (gene pyramiding), or expressing the protein only in tissues highly sensitive to damage (tissue-specific expression).

In conclusion, knowledge of how Bthost plants influences the life table parameters of S.litura can help one to understand the population dynamics and select for the proper measures in management of this insect. After laboratory studies, more attention should bedevoted to field experiments to obtain moreapplicable results in field conditions.

\section{Acknowledgements}

The authors would like to express their sincere thanks to $\mathrm{M} / \mathrm{s}$ Monsanto Seed Company, Hyderabad. For providing the seed for carry out present study.

\section{References}

Acharya, M.F., Vyas, H.J., Gedia, M.V and Patel, P.V. 2007. Life table, intrinsic rate of Increase and Age-specific Distribution of Helicoverpa armigera (Hub) on Cotton. Annals of Plant Protection Sciences.15 (2): 338-341.

Agrawal., A.A. 2001. Transgenerational consequences of plant responses to herbivory: an adaptive maternal effect? The American Naturalist. 157: 555 - 569.

Ali, A and Rizvi, P.Q. 2007. Age specific survival and fecundity table of Coccinellaseptempunctata L. (Coleoptera:Coccinellidae) on different aphid species, Annals of Plant Protection Sciences. 15: 329-334.

Andrewartha., H.G and Birch., L.C. 1954. The distribution and abundance of
animals.University of Chicago press. 782 . pp.

Atwal AS and Bain SS, 1974.Applied Animal Ecology (Ludhiana: Kalyani Publishers), New Delhi.

Awmack, C.S and Leather, S.R. 2002.Host plant quality and fecundity in herbivorous insects. Annual Review of Entomology. 47: 817-844.

Banna, A.A., Wahab, A.I., Akad, A.S and Amin, N.S. 2012.Efficiency of the bioagent Bacillus thuringensiskurstaki on the lesser cotton leafworm Spodoptera exigua (Hub).Egyptian Journal of Biological Sciences.5(2): 141-145.

Bilapate, G.G and Pawar, V.M. 1980. Life fecundity tables for Heliothis armigera (Hubner) (Lepidoptera: Noctuidae) on sorghum earhead. Proceedings of Indian National Science Academy. 89 (1): 69-73.

Bilapate, G.G. 1980. Investigation on H.armigera (Hub) in marathwada XIX field studies on larval and pupal parasite. Journal of Marathwada Agriculture University, Parbhani.

Birch., L.C. 1948. The intrinsic rate of natural increase of an insect population. Journal of Animal Ecology 17:15-26.

CABI. 2010. Crop protection compendium: global module. Commonwealth Agricultural Bureau International, Wallingford, UK. http://www.cabi.org /compendia/cpc/.

Coppel, H.C.,and Mertins, J.W. 1977. Biological insect pest suppression.Springer, Berlin, 327p.

Deevey, E. S., 1947, Life table for natural population of animals. Rev.Biol., 22: 283314.

Dhabi, M.R., Mehta, D.M., Patel, C.C and. Korat, D.M. 2009. Life table of diamondback moth, Plutellaxylostella (Linnaeus) on cabbage (Brassica oleraceae var. capitata L.).KarnatakaJournal of Agriculture Sciences. 22(2): (319-321).

Dhawan, A.K., Kamaldeep Singh and SarikaSaini. 2010. Laboratory evaluation of the effectiveness of Bollgard II cotton 
hybrids against tobacco caterpillar, Spodoptera litura Fab. (Lepidoptera: Noctuidae). Journal of Entomological Research. 34(4): 311-314.

Ehrlich, P. R. and D. D. Murphy. (1988) Plant chemistry and host range in insect herbivores. Ecology. 69: 908-909.

Gabre RM, Adham FK and Chi H, 2004. Life table of Chrysomya megacephala (Fabricius) (Diptera: Calliphoridae). ActaOecologica 27: 179-183.

Gedia, M.V., Vyas, H.J., Acharya, M.F and Patel, P.V. 2008.Studies on Life fecundity Tables of Spodoptera litura(Fabricius) on Groundnut.Annals of Plant Protection Sciences. 16(1): 74-77.

Gilliland, A., C.E. Chambers, E.J. Bone, and D.J. Ellar. 2002. Role of Bacillus thuringiensis Cry1 $\delta$-endotoxin binding in determining potency during Lepidopteran larval development. Applied Environment.Microbiology. 68:15091515.

Greenplate, J.T. 1999. Quantification of Bacillus thuringiensis insect control protein Cry 1Ac over time in Bollgard cotton fruit and terminals.Journal of Economic Entomology. 92: 1377-1383.

Hamilton, A.J., Endersby, N.M., Ridland, P.M., Zhang, J and Neal, M.2005. Effects of cultivar on oviposition preference, larval feeding and development time of diamond backmoth, Plutellaxylostella $(\mathrm{L}$. (Lepidoptera: Plutellidae) on some Brassica oleracea vegetable in victoria.Australian Journal of Entomology. 44:284-287.

Harcourt., D.G, 1969. The development of life tables in the study of natural insect populations. Annual Review of Entomology. 14: 175-191.

Hemchandra, O and Singh, T.K. 2003.Life Table, Rate of Increase and Stable-age Distribution of Plutellaxylostella (Linnaeus) on Cauliflower.Annals of Plant Protection Sciences. 11, 269-273.

Horwitz, A.R., Navon, A., Leviski, S., Yablonski, S and Niv, A. 2003. Contributions of Bt-transgenic cotton to the Israeli IRM strategy. Proceedings of World Cotton Research.Conference.-3, Cape Town, South Africa. pp. 1250-1253. Huffaker, C.B., Berryman, A.A and Laing, J.E.1984.Natural control of insect population. In: Huffakar, C.B., Rabb, R.L. (Eds).Ecological Entomology. Wiley, New York, PP.359-398.

Jeyakumar, P., Tanwar, R. K., Jat, M. C., Dhandapani, A., Bambawale, O., 2007, Spodoptera litura an emerging pest on $\mathrm{Bt}$ cotton (Cry1Ac) under North Indian conditions. Pesticide Research.Journal., 90: 197-200.

Kocourek,F., Havelka, J., Berankova, J and Jarosik,V.1994.Effect of temperature on Development Rate and Intrinsic Rate of increase of Aphis gossypii reared on greenhouse cucumber. EntomologiaExperimentaliset Applicata.71:59-64.

Kranthi, K.R. 2005. Insecticide Resistance: monitoring, mechanism and management manual. Published by the Director, Central Institute for Cotton Research, PB.No 2, Shankarnagar PO. Nagpur.

Kranthi, K.R., Naidu, S., Dharwad, C.S., Tatwawadi, A., Mate, K., Patil, E., Bharose, A.A., Behera, G.T., Wasaskar, R.M and Kranthi, S. 2005. Temporal and intra-plant variability of Cry1Ac expressing in Bt-cotton and its influence on the survival of cotton bollworm, Helicoverpa armigera (Hubner) (Noctuidae: Lepidoptera). Current Science. 89 (2): 291-298.

Kumar, R. 2005. Effect of Bt- cotton genotypes and $\mathrm{Bt}$ cry gene interaction on the performance of Helicoverpa armigera (Hubner). M.Sc. Thesis, Punjab Agricultural University, Ludhiana, India.

Le Roux, E.J. and other 1963. Population dynamics of agricultural and forest insect pests.Mem.Ent. Soc. Can. 32, 1-103.

Lee, K. P., D. Raubenheimer, S. T. Behmer and S. J. Simpson. (2003) A correlation between macronutrient balancing and insect host-plant range: evidence from the specialist caterpillar Spodoptera 
exempta(Walker). Journal of Insect Physiology. 49: 1161-1171.

Luttrell, R. G., Abbas, A., Youngh, S.Y and Kinghten, K.. 1998. Relative activity of commercial formulation of Bacillus thuringiensis against selected noctuid larvae (Noctuidae: Lepidoptera), Journal of Entomological Sciences, 33: 365-377.

McIntyre, G.S. and Gooding, R.H. 2000. Effects of maternal age on larval competitiveness in house flies.Heredity. 85: 480 - 489.

Morris, R.F and Miller, C.A. 1954. The development of life tables for the spruce bud worm. Canadian Journal of Zoology. 32: 283-301.

Murugan, M., Sathiah, N. Dhandapani, N., Rabindra, R. J. and Mohan, S. 2003. Laboratory assays on the role of Indian transgenic Bt cotton in the management of Helicoverpa armigera (Hunbner) (Noctuidae: Lepidoptera) Indian Journal of Plant Protection, 31(1):1-5.

Nava, D.E, Diez-Rodríguez G.I, Melo, M, Afonso A.P.S. 2008 Biologiaetabela de vida de fertilidade de Hypercompeindecisaemdieta artificial.PesqAgropec Bras 43:16651669

Patil, R.A., Mehta, D.M and Jat, B.L. 2014. Studies on Life Fecundity Tables of Spodoptera litura (Fabricius) on Tobacco NicotianatabacumLinnaeus.Entomology, Ornithology and Herpetology.3(1): 9-13.

Perlak, F.J., R.W. Deaton, T.A. Armstrong, R.L. Fuchs, S.R.Sims, J.T. Greenplate, and D.A. Fischhoff. 1990. Insect resistant cotton plants. Biotechnology 8:939-943.

Poole., P.W. 1974. An introduction to quantitative ecology. McGraw Hill, U.S.A. 111.

Price., P.W 1997. Insect Ecology. $3^{\text {rd }}$ edn. John Wiley \& Sons, Inc, New York.

Raubenheimer, D. and S. J. Simpson. 2003. Nutrient balancing in grasshoppers: behavioural and physiological correlates of diet breadth. Journal of Experimental Biology. 206: 1669-1681.
Razmjou J, Moharramipour S, Fathipour Y, Mirhoseini SZ. 2006. Effect of cotton cultivar on performance of Aphis gossypii (Homoptera: Aphididae) in Iran. Journal of Economic Entomology 99: 1820-1825.

Ricklefs, R.E. Miller and Miller, G.L. 2000. Ecology, $3^{\text {rd }}$ ed. Freeman and company. New York

Roff, D.A. 1992. The Evolution of Life Histories: Theory and Analysis. Chapman and Hall, New York. pp. 339-343.

Rosenthal, G. A. and M. R. Berenbaum (eds.). 1992.Herbivores: Their Interaction with Secondary Plant Metabolites, 2nd ed. Academic Press, San Diego, California.

Schoonhoven, L. M., T. Jermy and J. J. A. van Loon. (1998) Insect-Plant Biology: From Physiology to Evolution. Chapman and Hall, London.

Simpson, S. J., D. Raubenheimer, S. T. Behmer, A. Whitworth and G. A. Wright. 2002. A comparison of nutritional regulation in solitarious and gregarious phase nymphs of the desert locust, Schistocercagregaria. Journal ofExpeimental Biology. 205: 121-129.

Slobodkin, L.B. 1962. Growth and regulation in annual populations.184 pp, Holt, Rinehart and Winston, New York.

Somashekara, R. 2009. Evaluation of first and second generation $\mathrm{Bt}$ cotton genotypes for characterization of resistance to bollworms and tobacco caterpillar. M. Sc. (Ag.) Thesis, University of Agriculture Sciences, Dharwad, Karnataka (India).

Southhwood, T. R. E. 1981. Bionomics strategies and population parameters. In: May, (ed.) Theoretical Ecology: Principles and Applications, 2nd edn, Blackwell, Oxford, pp 30-52.

Southwood T.R.E., Henderson P.A. 2000.Ecological Methods.3rd ed Blackwell, Oxford, UK, 575 pp.

Storer, N.P, Babcock, J.M., Schlenz, M., Meade, T., Thompson, G.D. 2010 Discovery and characterization of field resistance to $\mathrm{Bt}$ maize: Spodoptera frugiperda(Lepidoptera: Noctuidae) in 
Puerto Rico. Journal of Economic Entomology 103: 1031-1038.

Suomela, J. (1996) Within-tree variability of mountain birch leaves causes variation in performance for Epirritaautumnatalarvae.Vegetation. 127: 77-83.

Syed,T.S and Abro,G.H. 2003. Effect of Brassica vegetable hosts on biology and life table parameters of PlutellaXylostella under laboratory condition.Pakisthan Journal of Biological Science.6: 18911896.

Tabashnik, B.E, Gassmann, A.J., Crowder, D.W., Carriere, Y. 2008. Insect resistance to $\mathrm{Bt}$ crops: evidence versus theory. Nature Biotechnology 26: 199-202.

Taghizadeh, R., Fathipour, Y. \&Kamali, K. (2008) Influence of temperature on life table parameters of Stethorusgilvifrons (Mulsant) (Coleoptera, Coccinellidae) fed on Tetranychusurticae Koch. Journal of Applied Entomology 132, 638-645.

Udikeri., S.S. 2006. Evaluation of new generation Bt genotypes, sustainability of Cry protein expression, computation of ETL, effect on aphid predators and development of IPM module for Bt cotton under rainfed conditions. M.Sc. Thesis. UAS, Dharwad, Karnataka.

Van Rensburg, J.B.J (2007) First report of field resistance by stem borer, Busseolafusca (Fuller) to Bt-transgenic maize. SouthAfrican Journal of Plant Soil 24: 147-151.

Verkerk, R.H.J and Wright, D.J. 1996. Multitropic interactions and Management of the Diamondback moth: a review. Bulletin of Entomological Research. 86:205-216.

Wan P., Zhang Y., Wu, K and Huang M. 2005. Seasonal expression profiles of insecticidal protein and control efficacy against Helicoverpa armigera for $\mathrm{Bt}$ cotton in the Yangtze River valley of China. Journal of Economic Entomology. 98: 195-201.

Woods, H.A.1999. Patterns and mechanisms of growth of fifth instar Manducasextacaterpillar following exposure in low or high protein food during early instar. Physiological Zoology. 72: 445-454.

\section{How to cite this article:}

Supriya, G.B., T.V.K. Singh, V. Sunitha, S.K. Vinod and Narendrareddy, C. 2018. Life Table Study of Tobacco Caterpillar Spodoptera litura (F.)(Noctuidae: Lepidoptera) on Different Bt Cotton Hybrids During 120-150 DAS (Days after Sowing). Int.J.Curr.Microbiol.App.Sci. 7(06): 2367-2386. doi: https://doi.org/10.20546/ijcmas.2018.706.283 